\title{
Non-intrusive uncertainty quantification in structural-acoustic systems using polynomial chaos expansion method
}

\author{
Mingjie Wang ${ }^{1}$, Qibai Huang ${ }^{1, a}$, Shande $\mathrm{Li}^{1}$, Lin $\mathrm{Li}^{1}$ and Zhifu Zhang ${ }^{1}$ \\ ${ }^{1}$ State Key Laboratory of Digital Manufacturing Equipment and Technology, Huazhong University of Science and \\ Technology, Wuhan 430074, P R China
}

\begin{abstract}
A framework of non-intrusive polynomial chaos expansion method (PC) was proposed to investigate the statistic characteristics of the response of structural-acoustic system containing random uncertainty. The PC method does not need to reformulate model equations, and the statistics of the response can be evaluated directly. The results show that compared to the direct Monte Carlo method (MCM) based on the original numerical model, the PC method is effective and more efficient.
\end{abstract}

\section{Introduction}

It is well known that traditional methods are conducted with to analysis the structural-acoustic system determined parameters. However, a structural-acoustic model inevitably contains some uncertain factors due to uncertain load conditions, uncertain materials and so on. Thus, it is necessary to investigate the structural-acoustic system considering uncertainties.

The MCM [1] is widely used due to its robust characteristic and the ease of implementation, while its main shortcoming is time consuming to obtain accurate results. Therefore, the MCM is usually employed to validate other approaches. Perturbation stochastic method is an effective method to deal with random uncertainty [2]. However, this method may be only limited for the problems with small uncertainty level of parameters. The polynomial chaos expansion method has been previously applied in uncertain analysis among different fields, such as fluid dynamics [3], structural dynamics [4] and acoustic fields [5,6] and so on. Sarkar et al. [4] employed the PC method to investigate the mid-frequency vibration of linear random systems with random uncertainty. LePage et al. [6] studied the sonar system performance in uncertain environments based on the PC method. The simulation results agree very well with the results estimated by MCM with less computational costs. The PC method is suitable for quantifying the uncertainty in the systems with uncertainties in initial conditions, inputs, or model parameters [7].

However, up to now, to the best of the authors' knowledge, the relevant research on the non-intrusive methods for quantifying the uncertainty in the structural-acoustic problems is still not reported. In this study, the PC method is adopted to study uncertain propagation in the structural-acoustic system containing random uncertainty. A 2D structural-acoustic model containing random uncertainty is investigated.

\footnotetext{
a Corresponding author : D201377175@hust.edu.cn
} 


\section{Theory and formulation}

\subsection{Structural-acoustic dynamic model containing random uncertainty}

The dynamic equation of a structural-acoustic system can be written as

$$
\mathbf{Z U}=\mathbf{f}
$$

where $\mathbf{f}$ denotes the force vector, $\mathbf{U}$ represents the response vector, $\mathbf{Z}$ denotes the structural-acoustic dynamic matrix, which can be given by

$$
\mathbf{Z}=-w^{2} \mathbf{M}+j w \mathbf{C}+\mathbf{K}
$$

where $\mathbf{K}, \mathbf{M}$ represent the structural-acoustic stiffness matrix, mass matrix, respectively. Rayleigh damping $\mathbf{C}=\alpha \mathbf{M}+\beta \mathbf{K}$ is considered. And $\alpha, \beta$ represent the Rayleigh damping coefficients. $w$ is the angle frequency.

Assume that the statistics information about uncertain parameters is known, and the uncertain parameters are treated as random variables. The vector of random variables $\xi=\left(\xi_{1}, \xi_{2}, \ldots, \xi_{n}\right)$ represents all the random parameters. The dynamic equation (Equation (1)) of the structural-acoustic system with random variables can be given by

$$
\mathbf{Z}(\xi) \mathbf{U}(\xi)=\mathbf{f}(\xi)
$$

Then the response of the structural-acoustic system considering random uncertainty can be calculated by the following equation

$$
\mathbf{U}(\xi)=\mathbf{Z}(\xi)^{-1} \mathbf{f}(\xi)
$$

\subsection{The polynomial chaos expansion method for random variables}

In this section, we assume that the structural-acoustic model contains normally distributed random variables. The response $U$ can be expressed by an expansion of multidimensional Hermite polynomials function $\xi=\left(\xi_{1}, \xi_{2}, \ldots, \xi_{n}\right)$ as follows

$$
U(\xi)=\sum_{k=0}^{\infty} u_{k} \psi_{k}(\xi)
$$

where $u_{k}$ represent the coefficients to be calculated, $\psi_{k}(\xi)$ are the Hermite polynomial chaos of order $k$. In the process of numerical computation, the terms given by Equation (5) must be truncated. Assume the number of the truncated terms is $s, U(\xi)$ can be approximated by

$$
U(\xi) \approx \sum_{k=0}^{s-1} u_{k} \psi_{k}(\xi)
$$

where the number of the truncated terms $s=(n+r) ! / n ! r !, n$ denotes the number of random dimensions, and $r$ represents the highest order of the polynomials $\psi$. The coefficients $u_{k}$ in Equation (6) can be obtained as follows

$$
u_{k}=\frac{\left\langle U(\xi), \psi_{k}\right\rangle}{\left\langle\psi_{k}^{2}\right\rangle}=\frac{1}{\left\langle\psi_{k}^{2}\right\rangle} \int U(\xi) \psi_{k}(\xi) w(\xi) d \xi
$$

The numerical integration in Equation (7) can be obtained by the tensor product quadrature method, i.e.

$$
\int U\left(\xi_{)}\right) \psi_{k}(\xi) w(\xi) d \xi \approx \sum_{q=1}^{N} U\left(\xi_{q}\right) \psi_{k}\left(\boldsymbol{\xi}_{q}\right) w\left(\boldsymbol{\xi}_{q}\right)
$$

where $N$ represents the total number of collocation points. The collocation points are produced by the roots of the Hermite polynomial of order $r+1$. Then the coefficient $u_{k}$ can be calculated via the following equation 


$$
u_{k} \approx \frac{1}{\left\langle\psi_{k}^{2}\right\rangle} \sum_{q=1}^{N} U\left(\xi_{q}\right) \psi_{k}\left(\xi_{q}\right) w\left(\xi_{q}\right)
$$

By varying $k$ from 0 to $\boldsymbol{S}-\mathbf{1}$, the coefficients vector can be obtained

$$
\begin{gathered}
{\left[u_{0}, \ldots, u_{s-1}\right]=\left[U\left(\xi_{1}\right), \ldots, U\left(\xi_{N}\right)\right] \mathbf{A}(\xi)} \\
\mathbf{A}(\xi)=\left[\begin{array}{ccc}
\mathrm{a}_{0}\left(\xi_{1}\right) & \cdots & \mathrm{a}_{s-1}\left(\xi_{1}\right) \\
\vdots & \ddots & \vdots \\
\mathrm{a}_{0}\left(\xi_{N}\right) & \cdots & \mathrm{a}_{s-1}\left(\xi_{N}\right)
\end{array}\right], \quad a_{k}\left(\xi_{q}\right)=\frac{1}{\left\langle\psi_{k}^{2}\right\rangle} \psi_{k}\left(\xi_{q}\right) w\left(\xi_{q}\right)
\end{gathered}
$$

After obtaining the coefficients vector, the mean $\mu$ and the standard variance $\sigma$ of $U(\xi)$ can then be calculated via the following equation

$$
\mu(U(\xi))=u_{0}, \quad \sigma(U(\xi))=\sqrt{\sum_{k=1}^{s-1} u_{k}^{2}\left\langle\psi_{k}^{2}\right\rangle}
$$

\section{Numerical study}

A 2D structural-acoustic problem taken from Wang and Huang [8] is adopted to investigate the performance of the PC method. The uncertain parameters, listed in Table 1, are assumed to be independent of each other.

Table 1. Values of the uncertain parameters

\begin{tabular}{llllll}
\hline parameters & $E_{a}(\mathrm{~m} / \mathrm{s})$ & $\rho_{a}\left(\mathrm{~kg} / \mathrm{m}^{3}\right)$ & $E_{s}\left(\mathrm{~kg} / \mathrm{m}^{3}\right)$ & $\rho_{s}(\mathrm{~Pa})$ \\
\hline \multirow{3}{*}{ values } & $\mu_{E_{a}}=7.0 \times 10^{10}$ & $\mu_{\rho_{a}}=2700$ & $\mu_{E_{s}}=2.11 \times 10^{11}$ & $\mu_{\rho_{s}}=7850$ \\
& $\sigma_{E_{a}}=7.0 \times 10^{8}$ & $\sigma_{\rho_{a}}=54$ & $\sigma_{E_{s}}=2.11 \times 10^{9}$ & $\sigma_{\rho_{s}}=157$ \\
\hline
\end{tabular}

First, we choose the order of PC as 2. Second, 70 samples are employed to evaluate the response of original system. Third, obtain the coefficient vector using Equation (10). Four, after building the PC model, Equation (12) is used to calculate expectation and standard variance of the response. Based on the original numerical model, the solutions yielded by the direct MCM with $10^{4}$ samples are regarded as the reference solutions.
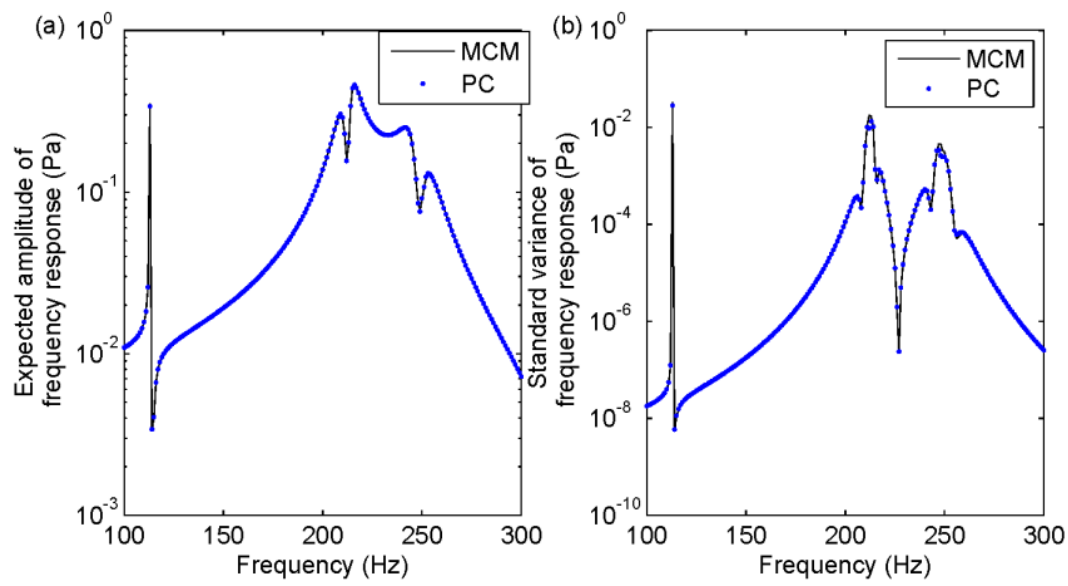

Figure 1. Statistic characteristics of response at node S: expectation (a) and standard variance (b) 
Table 2. The relative errors of statistic characteristics of the response at node $\mathrm{S}$

\begin{tabular}{cccccccc}
\hline \multirow{2}{*}{$\begin{array}{c}\text { Frequency } \\
(\mathrm{Hz})\end{array}$} & $\begin{array}{c}\mathrm{MCM} \\
(\mathrm{Pa})\end{array}$ & $\begin{array}{c}\text { PC } \\
(\mathrm{Pa})\end{array}$ & \begin{tabular}{c} 
Relative error \\
\cline { 6 - 7 } \cline { 6 - 7 }$(\%)$
\end{tabular} & & $\begin{array}{c}\text { MCM } \\
(\mathrm{Pa})\end{array}$ & $\begin{array}{c}\text { PC } \\
(\mathrm{Pa})\end{array}$ & $\begin{array}{c}\text { Relative error } \\
(\%)\end{array}$ \\
\hline 100 & $1.09 \mathrm{E}-02$ & $1.09 \mathrm{E}-02$ & 0.00 & & $1.78 \mathrm{E}-08$ & $1.75 \mathrm{E}-08$ & 2.15 \\
120 & $1.00 \mathrm{E}-02$ & $1.00 \mathrm{E}-02$ & 0.00 & & $2.62 \mathrm{E}-08$ & $2.55 \mathrm{E}-08$ & 2.37 \\
140 & $1.57 \mathrm{E}-02$ & $1.57 \mathrm{E}-02$ & 0.01 & & $9.06 \mathrm{E}-08$ & $8.83 \mathrm{E}-08$ & 2.48 \\
160 & $2.41 \mathrm{E}-02$ & $2.41 \mathrm{E}-02$ & 0.01 & & $3.92 \mathrm{E}-07$ & $3.82 \mathrm{E}-07$ & 2.64 \\
180 & $4.49 \mathrm{E}-02$ & $4.49 \mathrm{E}-02$ & 0.01 & & $3.18 \mathrm{E}-06$ & $3.09 \mathrm{E}-06$ & 2.86 \\
200 & $1.37 \mathrm{E}-01$ & $1.37 \mathrm{E}-01$ & 0.03 & & $1.13 \mathrm{E}-04$ & $1.09 \mathrm{E}-04$ & 3.21 \\
220 & $3.48 \mathrm{E}-01$ & $3.48 \mathrm{E}-01$ & -0.01 & & $4.80 \mathrm{E}-04$ & $4.72 \mathrm{E}-04$ & 1.71 \\
240 & $2.47 \mathrm{E}-01$ & $2.47 \mathrm{E}-01$ & 0.03 & & $5.19 \mathrm{E}-04$ & $5.09 \mathrm{E}-04$ & 1.97 \\
260 & $8.60 \mathrm{E}-02$ & $8.60 \mathrm{E}-02$ & 0.03 & & $6.42 \mathrm{E}-05$ & $6.17 \mathrm{E}-05$ & 3.96 \\
280 & $2.16 \mathrm{E}-02$ & $2.16 \mathrm{E}-02$ & -0.01 & & $2.69 \mathrm{E}-06$ & $2.65 \mathrm{E}-06$ & 1.57 \\
300 & $7.22 \mathrm{E}-03$ & $7.22 \mathrm{E}-03$ & -0.01 & & $2.52 \mathrm{E}-07$ & $2.48 \mathrm{E}-07$ & 1.75 \\
\hline
\end{tabular}

As shown in Figure 1 (a) and Figure 1 (b), the results obtained by the PC are similar to those obtained by MCM. To study the performance of the PC method in detail, the relative errors of statistic characteristics of response are listed in Table 2.From.

Table 2, we can see that the maximum relative error between PC and MCM is 3.96\%. Furthermore, the relative errors of standard variance are larger than the counterpart of expectation, which means that expectation are less sensitive to uncertain parameters compared to standard variance.

Table 3. Execution time of the PC and MCM

\begin{tabular}{|c|c|c|c|}
\hline MCM & \multicolumn{3}{|c|}{$\mathrm{PC}$} \\
\hline $\begin{array}{c}\text { Total } \\
\text { execution } \\
\text { time }(\mathrm{s})\end{array}$ & $\begin{array}{c}\text { Execution time } \\
\text { of determining } \\
\mathbf{U}(\mathrm{s})\end{array}$ & $\begin{array}{l}\text { Execution time of } \\
\text { determining statistic } \\
\text { characteristics (s) }\end{array}$ & $\begin{array}{l}\text { Total execution } \\
\text { time }(\mathrm{s})\end{array}$ \\
\hline $431,142.85$ & 3018.14 & 3.89 & 3022.03 \\
\hline
\end{tabular}

The execution time of the MCM and PC to calculate statistic characteristics of the response are shown in Table 3. We can see that the total execution time of the MCM method was considerably longer than that of the PC method. In fact, it only takes about $3022.03 \mathrm{~s}$ in the PC method, much less than the $431,142.85 \mathrm{~s}$ required by MCM. Furthermore, in the PC method, it takes the largest fraction of the execution time to obtain the response matrix $\mathbf{U}$, and the statistic characteristics of the response can be calculated by only extra $3.89 \mathrm{~s}$.

Based on the Equation (6), $10^{4} \mathrm{MCM}$ simulations are conducted to evaluate the PDF of the response. Based on the original numerical model, the direct MCM with total $10^{4}$ samples is regarded as the referenced solution. The response PDF for frequency $100 \mathrm{~Hz}, 200 \mathrm{~Hz}$ at node $\mathrm{S}$ using the direct MCM and the PC are plotted in Figure 2 (a) and Figure 2 (b), respectively. We can see that the overall shapes of the probability distribution mapped by the PC method practically coincided with the direct MCM results. 

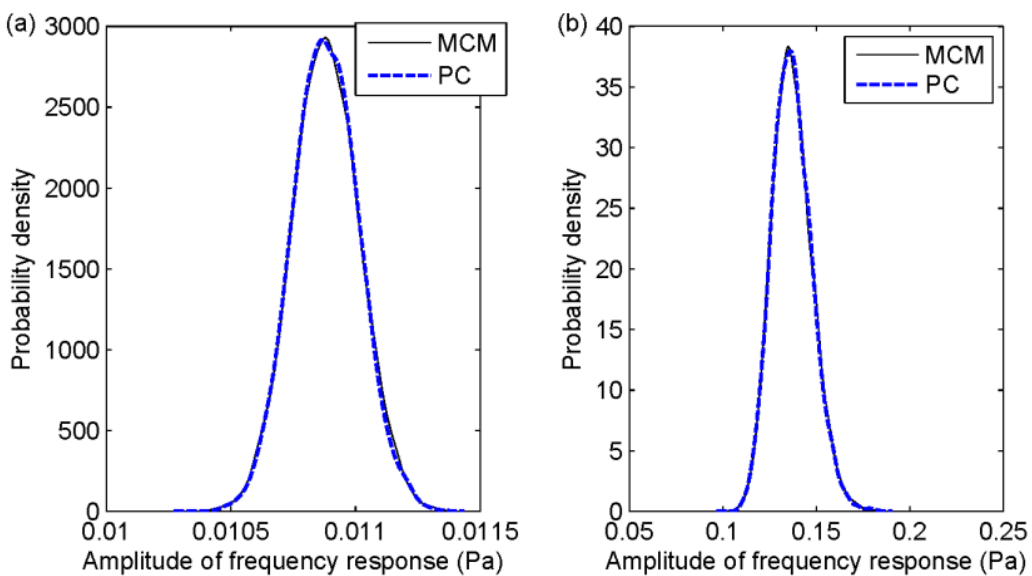

Figure 2. The response probability density of node S calculated by MCM and PC: (a) $100 \mathrm{~Hz}$, (b) $200 \mathrm{~Hz}$

Statistic characteristics of the response evaluated at nodes along the line $y=0.15 \mathrm{~m}$ for frequency $100 \mathrm{~Hz}, 200 \mathrm{~Hz}$ for are shown in Figure 3 and Figure 4, respectively. The results show that the results yielded by PC method match with solutions obtained by MCM perfectly.
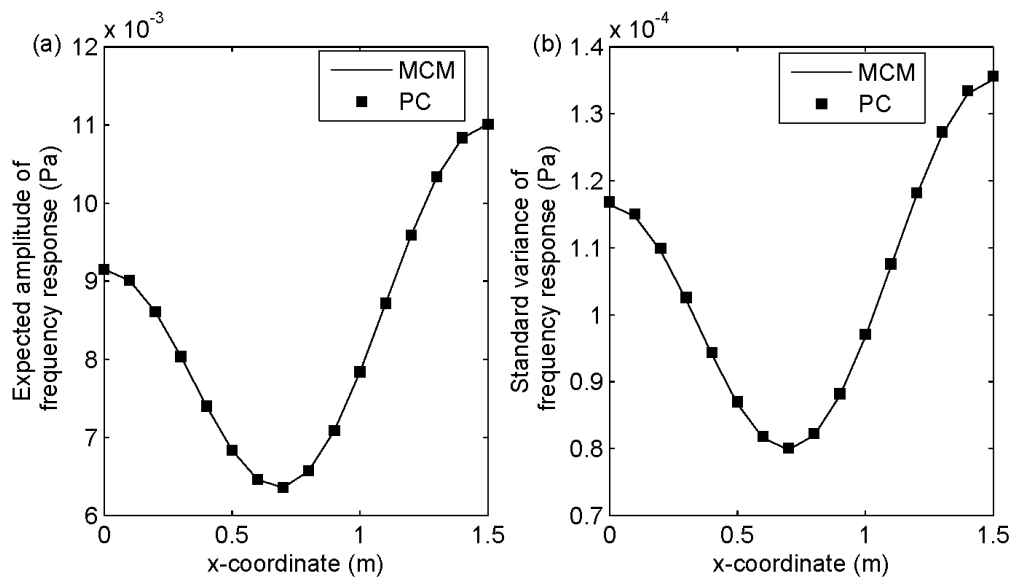

Figure 3. Statistic characteristics of the response along the line $y=0.15 m \quad(100 \mathrm{~Hz})$ : expectation (a) and standard variance (b) 

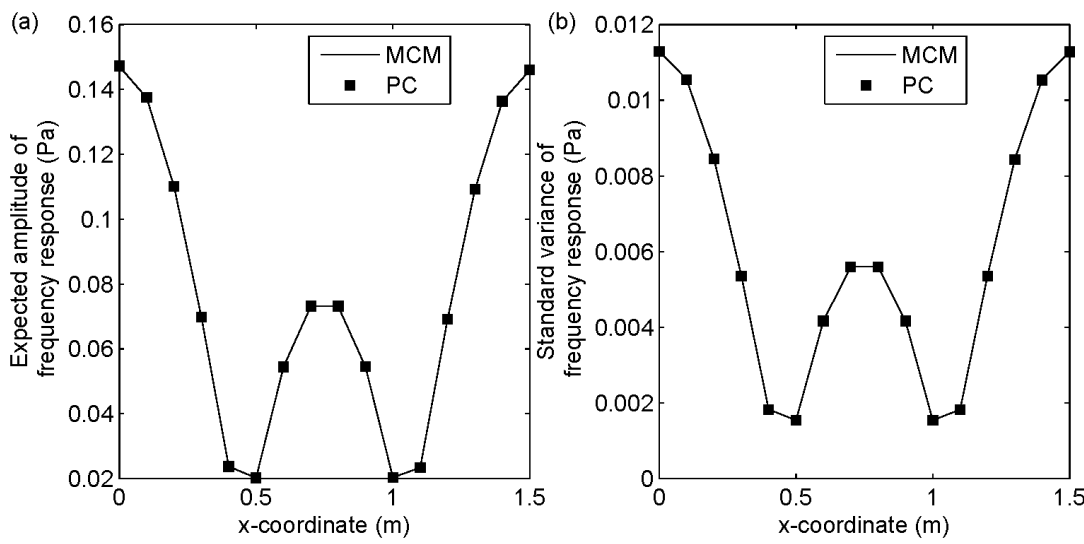

Figure 4. Statistic characteristics of the response along the line $y=0.15 m \quad(200 \mathrm{~Hz})$ : expectation (a) and standard variance (b)

\section{Conclusion}

The PC method is implemented to study uncertain propagation in the structural-acoustic problem containing random uncertainty. Based on the PC model, statistic characteristics of response of the structural-acoustic problem containing random uncertainty can be evaluated. The numerical results show that the PC method solutions match well with those yielded by the MCM. Furthermore, the computational effort of the PC method is significantly lower than that of the MCM.

\section{Acknowledgments}

The research was sponsored by the National Nature Science Foundation of China (No.51575201).

\section{References}

[1] M. Shinozuka. Monte Carlo solution of structural dynamics. Comput. Struct. 2 : 855-874(1972).

[2] G. I. Schuëller. Developments in stochastic structural mechanics. Arch. Appl. Mech. 75 : 755-773(2006).

[3] O. M. Knio, O. P. L. Maître. Uncertainty propagation in CFD using polynomial chaos decomposition. Fluid Dyn. Res. 38 : 616-640(2006).

[4] A. Sarkar, R. Ghanem. Mid-frequency structural dynamics with parameter uncertainty. Computer Methods in Applied Mechanics \& Engineering. 191 : 5499-5513(2002).

[5] M. Wang, Z. Wan, Q. Huang. A New Uncertain Analysis Method for the Prediction of Acoustic Field with Random and Interval Parameters. Shock \& Vibration. 2016 : 1-16(2016).

[6] K. D. Lepage. Estimation of Acoustic Propagation Uncertainty Through Polynomial Chaos Expansions. International Conference on Information Fusion. 2006:1-5(2006).

[7] Z. K. Nagy, R. D. Braatz. Distributional uncertainty analysis using power series and polynomial chaos expansions. J. Process Contr. 17 : 229-240(2007).

[8] M. Wang, Q. Huang. A new hybrid uncertain analysis method for structural-acoustic systems with random and interval parameters. Comput. Struct. $175: 15-28(2016)$. 